\title{
IMAGE FORMATION OF RADIALLY AND TEMPORALLY TRUNCATED BESSEL BEAMS
}

\author{
O. Rebane, M. Lõhmus, and P. Saari \\ University of Tartu, Institute of Physics, 142 Riia St, Tartu, 51014 Estonia \\ E-mail: ott.rebane@gmail.com
}

Received 16 October 2009; accepted 19 March 2010

\begin{abstract}
The Fourier-lens-formatted image of a radially truncated Bessel beam is theoretically calculated and compared with the experimentally measured results. A peculiarity - the weak central spot in the focused image of an apertured Bessel beam - has been recorded for the first time. Femtosecond-domain temporal evolution of the image of two types of superluminal localized wave packets - the so-called Bessel-X pulse and the focused X wave - is studied theoretically. For the Bessel-X pulse the aperture truncation leads to appearance of twin pulses in the image plane. In the case of the focused $X$ wave, the ring changes its colour revealing the full bandwidth of the ultrashort wave packet.
\end{abstract}

Keywords: Bessel beam, Bessel-X pulse, Fourier optics

PACS: $42.25 . F x, 42.25 . G y, 42.30 . K q$

\section{Introduction}

The existence of apparently non-diffracting and nonspreading ultrabroadband wave packets - the so-called localized waves [1] - in vacuum or linear media was theoretically predicted already in 1983 [2] and in the optical region experimentally proven afterwards [3]. Classification of the localized waves into subluminal, luminal, and superluminal ones - according to their group velocity ratio to the speed of light $c$ in vacuum - along with their characteristic properties has been presented in the review [4] and references therein.

Our aim in this article is to demonstrate what the intensity distribution in the back focal plane of an imaging device (lens, camera, etc) would look like if these superluminal light fields were to hit the device along its optical axis. In figurative words - we are investigating what a person would see if a faster-than-light wavefield was to fall straight into one's eye. The main wavefield under consideration is the Bessel-X pulse a. $\mathrm{k}$. a. Bessel-X wave (BXW) [3]. To simplify the understanding of the image formation of the $\mathrm{BXW}$, we first consider the image formation of a (monochromatic) Bessel beam. The reason for such approach is that the Bessel-X pulse is nothing but a specific superposition of Bessel beams of various frequencies. After studying the BXW we take a glimpse at another more general wavefield having superluminal group velocity: the focused-X-wave
(FXW). Throughout the text we consider only the farfield paraxial case and image formation is calculated by means of the Fourier transform only in the rear focal plane of the imaging device.

\section{Bessel beam}

A perfect Bessel beam can be thought of as consisting of an infinite number of plane waves, whose wave vectors $\mathbf{k}$ (distributed uniformly) in the momentum space lie on a conical surface that has a fixed cone vertex angle $2 \theta$. The result of such an axisymmetric superposition is a wavefield which has a complex wave field distribution that can be described by the zeroth order Bessel function of the first kind $\Psi_{\mathrm{X}}\left(r_{1}, t\right)=J_{0}\left(k_{\rho} r_{1}\right)$. $\exp \left(\mathrm{i} k_{z} z-\mathrm{i} \omega t\right)$ where the axial wave number $k_{z}$ is the component of $\mathbf{k}$ that is parallel to the optical axis and the radial wavenumber $k_{\rho}$ is perpendicular to it. Since a realistic Bessel beam cannot have an infinite aperture nor can the imaging device, we put a circular aperture stop with a known radius $r_{0}$ on the focusing lens that is used to find the image (Fourier transform) of this radially truncated Bessel function. Mathematically the operation and its result can be expressed as follows (the twodimensional Fourier transform of axisymmetric func- 


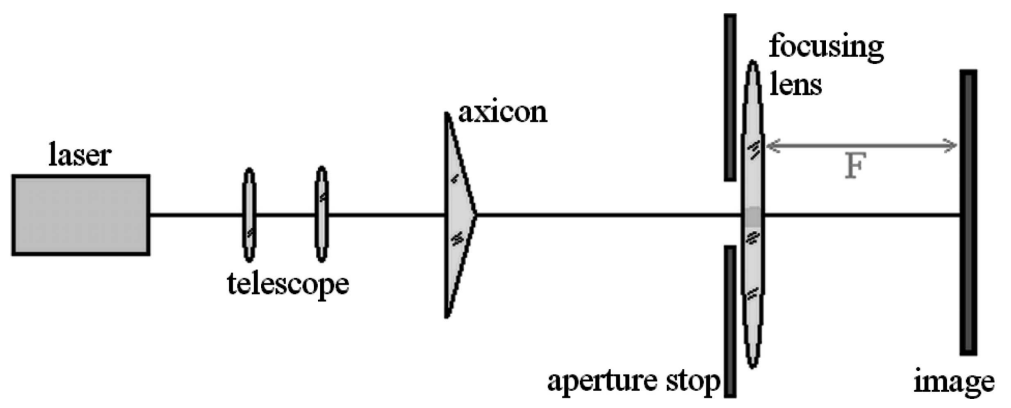

Fig. 1. The simplified scheme of set-up of the experiment to find the peculiar central intensity maximum. A HeNe laser at $633 \mathrm{~nm}$, the axicon's cone vertex angle $175 \mathrm{deg}$, and glass with refractive index 1.45 were used.
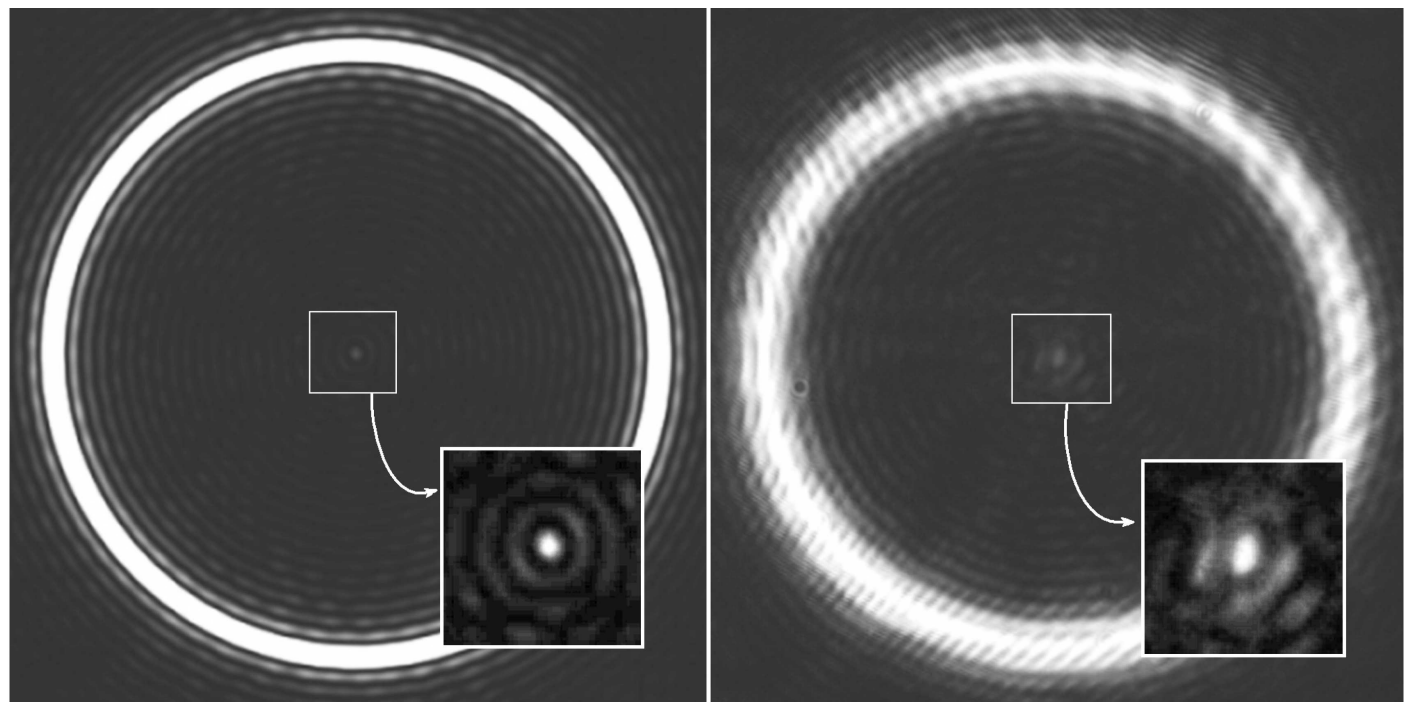

Fig. 2. The circular aperture stop with a radius of $0.7 \mathrm{~mm}$ - placed on the lens symmetrically with respect to the optical axis of the Bessel beam - produced a central maximum which can be seen in centres of both images. The image on the right is the experimental result and the image on the left is the theoretical prediction.

tions can be taken via the one-dimensional Hankel transform):

$$
\begin{aligned}
& \Psi_{\rho}(\rho, t)= \\
& 2 \pi \exp (-\mathrm{i} \omega t) \int_{0}^{r_{0}} r_{1} J_{0}\left(k_{\rho} r_{1}\right) J_{0}\left(\rho r_{1}\right) \mathrm{d} r_{1}= \\
& 2 \pi \exp (-\mathrm{i} \omega t) \frac{r_{0}}{k_{\rho}^{2}-\rho^{2}}\left[k_{\rho} J_{0}\left(\rho r_{0}\right) J_{1}\left(k_{\rho} r_{0}\right)-\right. \\
& \left.\quad-\rho J_{0}\left(k_{\rho} r_{0}\right) J_{1}\left(\rho r_{0}\right)\right],
\end{aligned}
$$

where $r_{1}$ denotes the radial coordinate in the front focal plane and we have set $z=0$ to the front focal plane. As can be seen from the result (1) by setting the radial coordinate $r_{3}=\rho F / k \Rightarrow r_{3} \sim \rho$ of the rear focal plane to zero, the intensity of the central spot depends on square of the Bessel function of the first order $J_{1}$. The relationship between $J_{0}$ and $J_{1}$ is that the derivative of $J_{0}$ is $-J_{1}$ which in our case means that if there is an intensity minimum at the edge of the aperture stop, then there is an intensity maximum in the centre of the image and vice versa. To verify the existence of such an effect, we carried out a simple experiment (Fig. 1) with two aperture stops - one of which should provoke a maximum in the centre of the image (Fig. 2) and the other one should not (result not shown in this article).

It might be noteworthy to say that to our best knowledge this peculiar central maximum has never before experimentally recorded on a picture - at least we found no publication on such an experiment. Mathematically this central spot has been derived in [5], but the central maximum in their experimental result is indistinguishable - presumably because they did not use a small enough aperture. The focusing properties of Bessel beams have also been theoretically studied in [6] and [7], but in these no particular emphasis has been put on this central spot nor do they have the relevant experimental part. From our experimental result (Fig. 2) 
we can see that there is a sufficiently good accordance between the theoretical and the experimental results. By "sufficiently good accordance" here we mean that even though the theoretical image has been computed for an ideal Bessel beam - i. e., we have not considered the Gaussian intensity profile before the axicon nor the axicon-specific-effect of intensity growth after the axicon, neither have we considered the effects of monochromatic light to our CCD-camera or the exact "noisy" shape of the aperture stop - still we can see a remarkable resemblance of the two images and most importantly we see the central maximum spot.

\section{Bessel-X pulse}

Whereas the Bessel beam could be described by a cone of fixed-length (monochromatic) wave vectors that form a static intensity distribution as well as a static image, the BXW is formed from an infinite number of ultrashort coherent pulses. The wave vectors of these pulses also lie uniformly on a conical surface with a fixed cone angle, but now the spectrum is polychromatic - as is the case with short pulses - which allows us to describe the Bessel-X pulse as a special superposition of Bessel beams. The BXW also is the simplest localized wave field which has its apex moving rigidly (the wave packet is propagation-invariant) and superluminally (in the case of BXW the intensity distribution as well as the wave function or EM-field distribution have these properties) along the optical axis of the pulse.

Keeping in mind that the BXW is a superposition $(S(k)$ representing the spectrum of it) of many monochromatic Bessel beams,

$$
\begin{aligned}
& \Psi_{\mathrm{bxw}}\left(r_{3}, t\right)=\int_{-\infty}^{\infty} \Psi_{\rho}\left(r_{3}, k, t\right) S(k) \mathrm{d} k= \\
& =\frac{r_{0}}{F^{2} \sin ^{2} \theta-r_{3}^{2}} \int_{-\infty}^{\infty} \frac{\exp (-\mathrm{i} k c t)}{\mathrm{i}} \\
& \times \exp \left[\mathrm{i} k\left(2 F+\frac{r_{3}^{2}}{2 F}\right)\right] S(k)\left[F \sin \theta J_{0}\left(\frac{r_{3} k}{F} r_{0}\right)\right. \\
& \left.\times J_{1}\left(k_{\rho} r_{0}\right)-r_{3} J_{0}\left(k_{\rho} r_{0}\right) J_{1}\left(\frac{r_{3} k}{F} r_{0}\right)\right] \mathrm{d} k,
\end{aligned}
$$

it is possible to derive some properties of the image of the Bessel-X pulse intuitively. Namely - by changing the monochromatic Bessel beam's colour, but not the cone angle, we get different width to the Bessel function hitting the lens, which in turn means that for some frequencies - in fact for most of them - the image has to have a central intensity spot. This means that the image of a BXW always has to show a central intensity spot. Since all the component Bessel beams of different frequencies produce a ring in the image, it also has to have a very intense ring. Here we have not considered relation between the phases of different Bessel beam components - therefore we do not know the temporal behaviour of the image. The time-dependence will become clear if we approximate the complex electric field distribution of ultrashort optical pulses, that the BXW consists of, with the first derivative of the Dirac delta function $A \delta^{\prime}(z)$ (in time domain) and take the Fourier transform of such a wave field.

After some cumbersome derivation ${ }^{1}$, using the circumstance that far from the argument's origin the function $J_{0}$ can be approximated by the cos function and $J_{1}$ with the sin function, we get the approximate wave field near the intense ring to be

$$
\begin{aligned}
& \Psi_{\text {bxw_far }}\left(r_{3}, t\right)=\frac{4 \cos \theta}{\left(F^{2} \theta^{2}-r_{3}^{2}\right)} \sqrt{\frac{F}{r_{3} \theta}} \\
& \times \int_{-\infty}^{\frac{1}{\cos \theta}\left(2 F+\frac{r_{3}^{2}}{2 F}-t c\right)} s(z) * g(z) \mathrm{d} z= \\
& =\frac{-1 \cos \theta}{\left(F \theta+r_{3}\right)} \sqrt{\frac{F}{r_{3} \theta}} A \cos \theta\left[\delta \left(2 F+\frac{r_{3}^{2}}{2 F}+\frac{r_{0}}{F} r_{3}\right.\right. \\
& \left.\left.+r_{0} \theta-t c\right)+\delta\left(2 F+\frac{r_{3}^{2}}{2 F}-\frac{r_{0}}{F} r_{3}-r_{0} \theta-t c\right)\right] \\
& +\frac{1 \cos \theta}{\mathrm{i}\left(r_{3}-F \theta\right)} \sqrt{\frac{F}{r_{3} \theta}} A \cos \theta\left[\delta \left(2 F+\frac{r_{3}^{2}}{2 F}+\frac{r_{0}}{F} r_{3}\right.\right. \\
& \left.\left.-r_{0} \theta-t c\right)-\delta\left(2 F+\frac{r_{3}^{2}}{2 F}-\frac{r_{0}}{F} r_{3}+r_{0} \theta-t c\right)\right] .
\end{aligned}
$$

Here $s(z)=A \delta^{\prime}(z)$ represents the ultrashort pulse in time domain and $g(z)$ is the backward Fourier transform of the $\sin -\cos$ approximation of (1). The asterisk denotes convolution operation as usual, $F$ is the focal length of the focusing lens, $r_{0}$ is the radius of the aperture stop, and $r_{3}$ is the radial coordinate in the back focal plane.

\footnotetext{
${ }^{1}$ To make the equations shorter, the sine and tangent of a paraxial angle are substituted with the angle.
} 
It gets even more complex in the centre of the image (here we denote $(-t c+2 F) / \cos \theta \equiv z$ ):

$$
\begin{aligned}
& \Psi_{\text {bxw_cen }}(0, t)=\int_{-\infty}^{\infty} \Psi_{3}(0, k, t) S(k) \mathrm{d} k= \\
& =\frac{2 \pi r_{0}}{\mathrm{i} F \theta} \frac{1}{2 \pi} \int_{-\infty}^{\infty} S_{z}\left(k_{z}\right) J_{1}\left(k \sin \theta r_{0}\right) \\
& \quad \times \exp \left[\mathrm{i} k_{z} \frac{1}{\cos \theta}(-t c+2 F)\right] \mathrm{d} k_{z} \\
& =\frac{2 \cos \theta}{F \theta^{2}} A \delta^{\prime}(z) * \frac{z r e c t\left(\frac{z}{2 r_{0} \theta}\right)}{\sqrt{\left(r_{0} \theta\right)^{2}-z^{2}}}= \\
& =\frac{2 A \cos \theta}{F \theta^{2}\left[\left(r_{0} \theta\right)^{2}-z^{2}\right]^{\frac{3}{2}}\left\{\left(r_{0} \theta\right)^{2} r e c t\left(\frac{z}{2 r_{0} \theta}\right)\right.} \\
& +\left(r_{0} \theta\right)^{2} z\left[\delta\left(z+r_{0} \theta\right)-\delta\left(z-r_{0} \theta\right)\right] \\
& \left.-z^{3}\left[\delta\left(z+r_{0} \theta\right)-\delta\left(z-r_{0} \theta\right)\right]\right\} .
\end{aligned}
$$

By examining the time differences between these flashes, the results can be interpreted in the way that the so-called boundary waves [8] (in the sense of boundaries acting as if they were the secondary sources according to the Huygens-Fresnel principle) appear from the edges of the aperture stop - Fig. 3 parts 1 and 3 and contribute to the temporally resolved image. If we were to look at the ring's position (Eq. (3)) and we could measure the temporal behaviour of such fast effects, three time instances could clearly be resolved: first we would see the boundary wave that appears when the BXW's two-sided "light cone" first meets the aperture stop (Fig. 3 part 1), then we would see the main field converged by the lens, and finally we would see the second boundary wave appear; this last one is formed when the light cone's tail hits the aperture stop (Fig. 3 part 3). We are interested in the central spot of the image: from the theory we can conclude that at two instants the central spot "shines out" when the intensity is particularly high (Eq. (4)). Both of these central spot flashes (Fig. 3 parts 6 and 7) can be interpreted as a sum of boundary waves from the geometric shadow region diffracting to centre of the image. Between these pulses - the spots that we predicted from the intuitive model - the intensity is very small but oddly not zero.

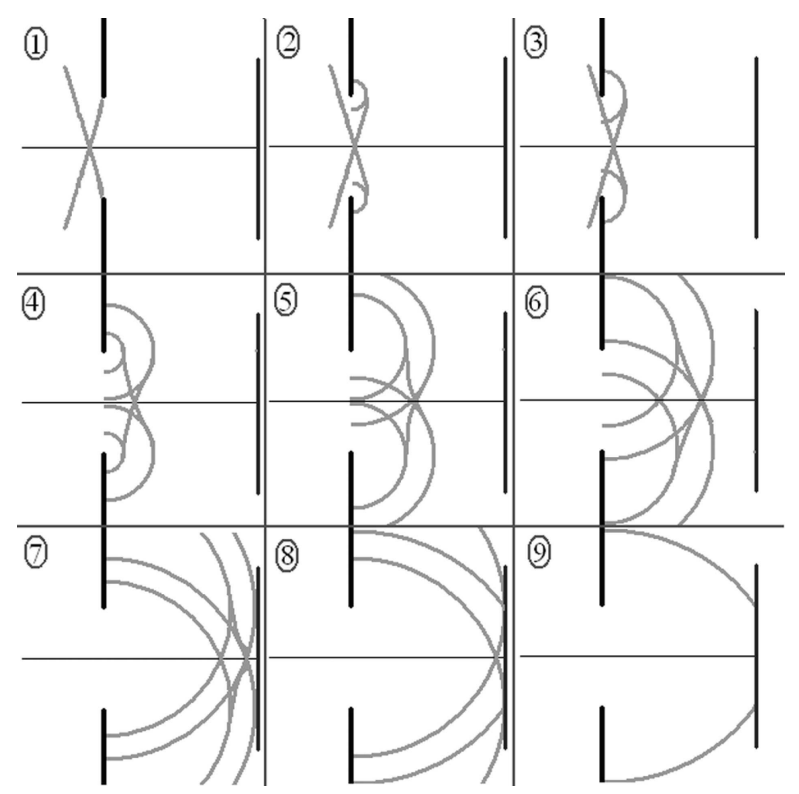

Fig. 3. An illustration to our interpretation of the time-dependent intensity distribution in the central cross-section of the imaging device. The focusing lens in the centre of the aperture stop is not depicted nor are the different intensities distinguishable. The nine subpictures are enumerated according to their order of occurrence. In part 1 the double light cone first hits the aperture stop and produces a secondary wave (1st boundary wave) in the shadow region. In part 3 the tail of the light cone hits the aperture stop and the second boundary wave is formed. In parts 6 and 7 the moving central spots can clearly be distinguished. In part 7 the boundary wave reaches the ring's position, part 8 shows the arrival of the intense converged geometrical field, and part 9 depicts the arrival of the second boundary wave to the Fourier plane in the ring's position.

Intensity differences between the ring and the boundary waves are not depicted on Fig. 3 but of course the converging geometrical waves are much more intense (Eq. (3)) than the boundary waves. The X-shaped crosssection of the converging geometrical waves - a socalled Bessel pulse [9] that is most clearly depicted on Fig. 3 parts 4 and 5 - and its central maximum's accelerating motion have already been directly recorded [9] using a novel technique called SEA TADPOLE [10].

\section{Focused X wave}

Just for the purpose of exploring the theoretically derived images of the superluminal localized wave fields we introduce the FXW and its image. As already mentioned, a generalized localized wave field can be synthesized [11] which has its central intensity maximum moving at superluminal group velocity - the Focused$\mathrm{X}$-Wave (FXW, the term proposed in [12]). To avoid confusion, we note that the word focused here has nothing to do with optical focusing - it simply describes the shape of the wave function of the FXW. The FXW can 
also be described as a superpostion of Bessel beams, but with another dispersion relation between the axial wavenumber and the frequency, which makes the angle $\theta$ frequency-dependent [11]. Here the Bessel beams' cone vertex angles as well as $\mathbf{k}$-vector lengths vary - they are following a hyperbolic relation between the wave number's axial component $k_{z}$ and the radial component $k_{\rho}$. The longer wave vectors that have a larger angle between the optical axis and themselves are weighed down by some spectral function like falling exponent.

When calculating the Fourier image of such a wave field - for simplicity the aperture has been taken infinitely wide - we found that the result should be a static picture of a concentric "rainbow". This result is of course a purely theoretical one and at first glance also illogical - there should be some temporal dependence since it is a pulse - but the ideas of infinite aperture and a perfect lens are also quite illogical so the static rainbow hypothesis here might as well be true. These problems we left to a future study.

\section{Conclusions}

We have investigated the image formation of Bessel beams and found a peculiar aperture-dependent spot in the centre of the image. We have also experimentally verified the existence of such central spot for the first time. We have carried out calculations of the spatiotemporal properties of the image of a Bessel-X pulse and we found out that the resultant time-dependent image can most easily be interpreted by the notion of boundary wave diffraction. As a result, which needs further study, we considered the oddities found in an infiniteaperture image of a FXW light field: namely the apparently static intensity distribution for a pulsed wave field such as FXW.

\section{References}

[1] Localized Waves: Theory and Applications, ed. H.E. Hernandez-Figueroa, M. Zamboni-Rached, and E. Recami (Wiley, New Jersey, 2008).

[2] J.N. Brittingham, Focus wave modes in homogeneous Maxwell equations: Transverse electric mode, J. Appl. Phys. 54, 1179 (1983).

[3] P. Saari and K. Reivelt, Evidence of X-shaped propagation-invariant localized light waves, Phys. Rev. Lett. 79, 4135 (1997).

[4] P. Saari and K. Reivelt, Generation and classification of localized waves by Lorentz transformations in Fourier space, Phys. Rev. E 69, 036612 (2004).

[5] B. Lü, W. Huang, and B. Zhang, Fraunhofer diffraction of a Bessel beam focused by an aperture lens, Opt. Commun. 119, 6 (1995).

[6] B. Lü, W. Huang, B. Zhang, F. Kong, and Q. Zhai, Focusing properties of Bessel beams, Opt. Commun. 131, 223 (1996).

[7] B. Lü and W. Huang, Three-dimensional intensity distribution of focused Bessel-Gauss beams, J. Mod. Opt. 43, 509 (1996).

[8] Z.L. Horvath and Zs. Bor, Diffraction of short pulses with boundary diffraction wave theory, Phys. Rev. E 63, 026601 (2001).

[9] H. Valtna-Lukner, P. Bowlan, M. Lõhmus, P. Piksarv, R. Trebino, and P. Saari, Direct spatiotemporal measurements of accelerating ultrashort Bessel-type light bullets, Opt. Express 17, 14948 (2009).

[10] P. Bowlan, U. Fuchs, R. Trebino, and U.D. Zeitner, Measuring the spatiotemporal electric field of tightly focused ultrashort pulses with sub-micron spatial resolution, Opt. Express 16, 13663 (2008).

[11] H. Valtna, K. Reivelt, and P. Saari, Methods for generating wideband localized waves of superluminal group velocity, Opt. Commun. 278, 1 (2007).

[12] I. Besieris, M. Abdel-Rahman, and A. Shaarawi, Two fundamental representations of localized pulse solutions to the scalar wave equation, Prog. Electromagn. Res. 19, 1 (1998). 


\title{
LAIKE IR ERDVĖJE APRIBOTŲ BESELIO PLUOŠTŲ VAIZDO FORMAVIMAS
}

\author{
O. Rebane, M. Lõhmus, P. Saari
}

Tartu universiteto Fizikos institutas, Tartu, Estija

\section{Santrauka}

Lęšio suformuotas radialiai apriboto Beselio pluošto Furjè vaizdas yra apskaičiuotas teoriškai ir palygintas su eksperimentiniais matavimo rezultatais. Jo ypatybè - silpna centrinè dėmé apertūrą peréjusio Beselio pluošto fokusuotame vaizde - užfiksuota pirmą kartą. Teoriškai tiriama dviejų tipų šviesos lokalizuotų bangų pa- ketu - vadinamojo Beselio X impulso ir fokusuotosios $\mathrm{X}$ bangos atvaizdo evoliucija femtosekundiniame diapazone. Beselio X impulso apertūrinis apribojimas sukelia dvigubų impulsų atsiradimą atvaizdo plokštumoje. Fokusuotosios X bangos atveju žiedas keičia spalvą, parodydamas visą ultratrumpujjų bangų paketo spektrini ploti. 